\title{
SI - Trends in Thermodynamic Stability of Ultrathin Supported Oxide Films
}

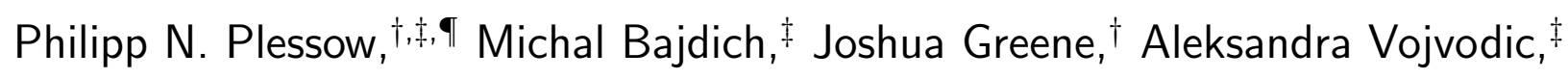 \\ and Frank Abild-Pedersen*,‡ \\ $\dagger S U N C A T$ Center for Interface Science and Catalysis, Department of Chemical \\ Engineering, Stanford University, Stanford, CA 94305, USA. \\ $\ddagger S U N C A T$ Center for Interface Science and Catalysis, SLAC National Accelerator \\ Laboratory, Menlo Park, CA 94025, USA. \\ \Current address: Institute of Catalysis Research and Technology (IKFT), \\ Hermann-von-Helmholtz-Platz 1, D-76344 Eggenstein-Leopoldshafen, Germany. \\ E-mail: abild@slac.stanford.edu
}




\section{Reference Energies}

The reference energy of oxygen was obtained from water and hydrogen by correcting the experimental reaction energy by the zero point energies:

$$
E(\mathrm{O})=E\left(\mathrm{H}_{2} \mathrm{O}\right)-E\left(\mathrm{H}_{2}\right)+2.75 \mathrm{eV}
$$

The experimental formation enthalpies of oxides below were obtained from the literature. ${ }^{1,2}$

Table S1: Oxides and formation energies. Metals have also been included with a formation energy of 0 .

\begin{tabular}{|c|c|}
\hline $\mathrm{Ni}($ bulk$)$ & 0.00 \\
\hline $\mathrm{Ni}_{2} \mathrm{O}_{3}$ & -5.07 \\
\hline $\mathrm{Co}($ bulk $)$ & 0.00 \\
\hline $\mathrm{Co}_{3} \mathrm{O}_{4}$ & -9.23 \\
\hline $\mathrm{CoO}$ & -2.47 \\
\hline $\mathrm{MnO}_{2}$ & -5.39 \\
\hline $\mathrm{Mn}_{3} \mathrm{O}_{4}$ & -14.38 \\
\hline $\operatorname{Mn}($ bulk$)$ & 0.00 \\
\hline $\mathrm{Mn}_{2} \mathrm{O}_{3}$ & -9.94 \\
\hline $\mathrm{MnO}$ & -3.99 \\
\hline $\mathrm{TiO}_{2}$ & -9.78 \\
\hline Ti(bulk) & 0.00 \\
\hline $\mathrm{Ti}_{2} \mathrm{O}_{3}$ & -15.76 \\
\hline $\mathrm{Ti}_{3} \mathrm{O}_{5}$ & -25.49 \\
\hline $\mathrm{Fe}_{3} \mathrm{O}_{4}$ & -11.59 \\
\hline $\mathrm{Fe}(\mathrm{bulk})$ & 0.00 \\
\hline $\mathrm{Fe}_{2} \mathrm{O}_{3}$ & -8.54 \\
\hline $\mathrm{FeO}$ & -2.82 \\
\hline $\mathrm{V}_{2} \mathrm{O}_{5}$ & -16.07 \\
\hline $\mathrm{V}$ (bulk) & 0.00 \\
\hline $\mathrm{V}_{2} \mathrm{O}_{3}$ & -12.63 \\
\hline $\mathrm{VO}$ & -4.48 \\
\hline $\mathrm{V}_{3} \mathrm{O}_{5}$ & -20.03 \\
\hline $\mathrm{CrO}_{2}$ & -6.20 \\
\hline $\mathrm{Cr}_{3} \mathrm{O}_{4}$ & -15.87 \\
\hline Cr(bulk) & 0.00 \\
\hline $\mathrm{Cr}_{2} \mathrm{O}_{3}$ & -11.81 \\
\hline
\end{tabular}




\section{Formation energies}

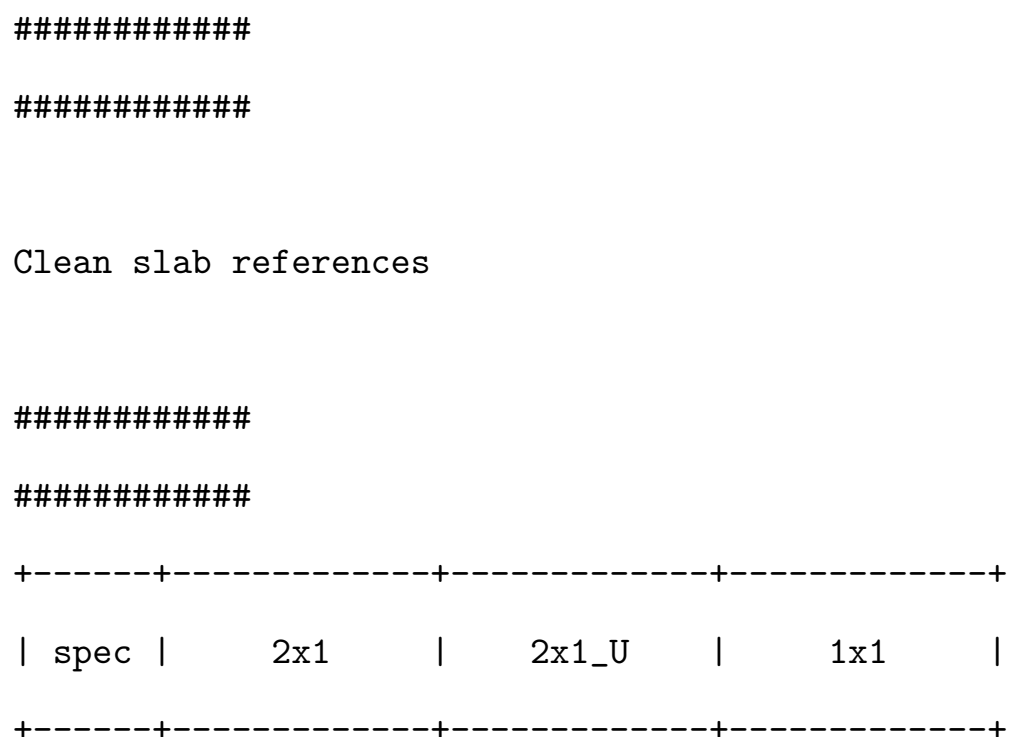

| $\mathrm{Ni}|-10944.1340|-10934.2334 \mid-5471.9654$ |

| Co | None | None | -4356.3786 |

| Ag | $-10331.7540|-10331.7540|-5165.8768$ |

| Pt | $-7907.9113|-7907.9113|-3953.9556$ |

| $\mathrm{Ru}|-5463.1100|-5463.1100 \mid-2731.5548$ |

| $\mathrm{Rh}|-25373.3714|-25373.3714 \mid-12686.6855$ |

| Ir | $\quad-6748.7040|-6748.7040|-3374.3520$ |

| $\mathrm{Au}|-9294.2580|-9294.2580 \mid-4647.1289$ |

\begin{tabular}{ll|l|l|l|l|}
\hline & $P d$ & $\mid$ & -8570.8424 & -8570.8424 & -4285.4211
\end{tabular} \mid

| 0 s | $-7268.7842|-7268.7842|-3634.3920$ |

| $\mathrm{Cu}|-12776.5350|-12776.5350 \mid-6388.2666$ |

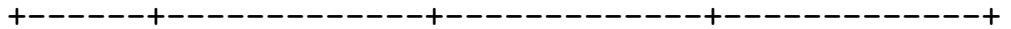

\#\#\#\#\#\#\#\#\#\#\#

\#\#\#\#\#\#\#\#\#\#\#

\#\#\#\#\#\#\#\#\#\#\#

\#\#\#\#\#\#\#\#\#\#\# 
bulk references

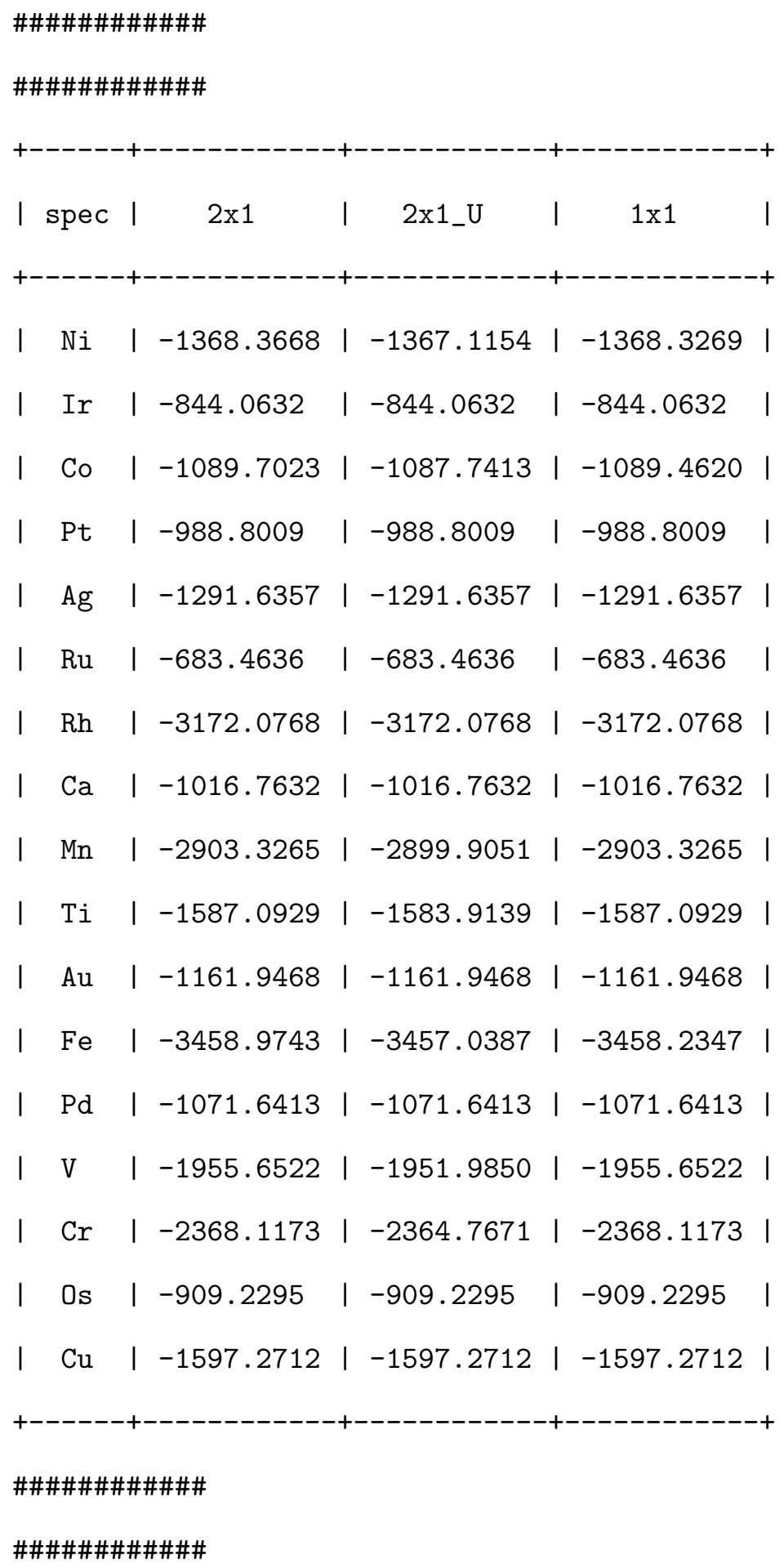




\section{\#\#\#\#\#\#\#\#\#\#\#\# \\ \#\#\#\#\#\#\#\#\#\#\#\#}

Adsorption energies. spec1 is the support, spec2 is the adsorbate.

\section{\#\#\#\#\#\#\#\#\#\#\#\#}

\#\#\#\#\#\#\#\#\#\#\#\#

\begin{tabular}{|c|c|c|c|c|c|c|c|c|c|}
\hline & pec1 & I & pec2 & I & $2 \times 1$ & I & $2 \mathrm{x} 1_{-} \mathrm{U}$ & I & $1 \times 1$ \\
\hline & $\mathrm{Ni}$ & I & $\mathrm{Ni}$ & I & None & I & 0.5005 & I & 1.2624 \\
\hline & $\mathrm{Ni}$ & I & Co & | & 1.1997 & I & None & 1 & 1.9585 \\
\hline & $\mathrm{Ni}$ & I & $\mathrm{Mn}$ & | & 0.2413 & I & None & I & 2.7551 \\
\hline & $\mathrm{Ni}$ & I & $\mathrm{Ti}$ & I & 1.9399 & I & None & I & 1.9975 \\
\hline & $\mathrm{Ni}$ & I & $\mathrm{Fe}$ & | & None & I & -0.8212 & I & 1.3957 \\
\hline & $\mathrm{Ni}$ & I & V & | & None & I & None & I & 1.8649 \\
\hline & $\mathrm{Ni}$ & I & $\mathrm{Cr}$ & I & None & I & -1.7027 & I & 2.7271 \\
\hline & Co & I & $\mathrm{Ni}$ & I & None & I & None & I & 0.9853 \\
\hline & Co & I & Co & I & None & I & None & I & 1.5559 \\
\hline & Co & I & $\mathrm{Mn}$ & I & None & | & None & I & 1.9456 \\
\hline & Co & I & $\mathrm{Ti}$ & I & None & I & None & I & 1.7576 \\
\hline & Co & I & $\mathrm{Fe}$ & I & None & I & None & I & 0.8762 \\
\hline & Co & I & V & I & None & I & None & I & 1.1963 \\
\hline & Co & I & $\mathrm{Cr}$ & I & None & I & None & I & 1.8304 \\
\hline & $\mathrm{Ag}$ & I & $\mathrm{Ni}$ & I & 2.3260 & I & 2.1027 & I & 2.2856 \\
\hline & $\mathrm{Ag}$ & I & Co & I & 2.8969 & I & 1.4245 & I & 3.4514 \\
\hline & $\mathrm{Ag}$ & I & $\mathrm{Mn}$ & I & 1.8364 & I & -1.1815 & I & 5.2816 \\
\hline & $\mathrm{Ag}$ & I & $\mathrm{Ti}$ & I & 5.0767 & I & 2.8520 & I & 5.5591 \\
\hline & $\mathrm{Ag}$ & 1 & $\mathrm{Fe}$ & & 2.0070 & & 0.4696 & 1 & 3.1934 \\
\hline
\end{tabular}




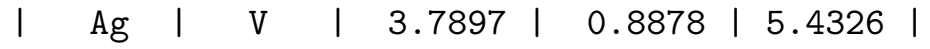

$$
\begin{aligned}
& \text { | } \mathrm{Ag}|\mathrm{Cr}| 2.4485 \text { | }-0.6916 \text { | } 5.8382 \text { | } \\
& \text { | Pt | Ni | } 1.0349 \text { | } 0.7534 \mid 1.0330 \text { | } \\
& \begin{array}{lllllllll}
\text { | Pt | Co | } & 1.3451 & 0.4203 & 1.6427 & \text { | }
\end{array} \\
& \text { | Pt | Mn | }-0.0988 \text { | }-2.8113 \text { | } 2.3175 \text { | } \\
& \text { | Pt | Ti | } 1.2217 \text { | } 0.8960 \mid 1.2223 \text { | } \\
& \text { | Pt | Fe | } 0.2648|-0.6283| 1.0989 \text { | } \\
& \text { | Pt | V | } 1.2059 \text { | }-0.2972 \mid 1.2379 \text { | } \\
& \text { | Pt | Cr | } 0.9077 \text { | }-1.5618 \text { | } 2.1932 \text { | } \\
& \begin{array}{lllllllllll} 
& \mathrm{Ru} & \mathrm{Ni} & 0.8028 & 0.8353 & 0.7626 & \text { | }
\end{array} \\
& \text { | } \mathrm{Ru}|\mathrm{Co}| 1.1894 \text { | } 0.6923 \text { | } 1.2261 \text { | } \\
& \text { | } \mathrm{Ru}|\mathrm{Mn}| 0.2170 \text { | }-2.4619 \text { | } 1.6519 \text { | } \\
& \text { | } \mathrm{Ru}|\mathrm{Ti}| 1.6662 \text { | } 1.1073 \text { | } 1.6660 \text { | } \\
& \text { | } \mathrm{Ru} \quad|\quad \mathrm{Fe} \quad| \quad 0.2545 \quad|\quad-0.6884| 0.5079 \text { | } \\
& \text { | } \mathrm{Ru}|\mathrm{V} \quad| \begin{array}{lllll} 
& 1.1641 & -0.2238 & 1.1644
\end{array} \\
& \text { | } \mathrm{Ru} \quad \mathrm{Cr} \quad 1.1187 \text { | }-1.5192 \text { | } 1.7245 \text { | } \\
& \text { | } \mathrm{Rh}|\mathrm{Ni}| 1.0931 \text { | } 1.0860 \text { | } 1.0524 \text { | } \\
& \text { | } \mathrm{Rh} \mathrm{|} \text { Co | } 1.4593 \text { | } 0.6015 \text { | } 1.5913 \text { | } \\
& \text { | } \quad \text { Rh | Mn | } 0.3200 \text { | }-2.3937 \text { | } 2.0269 \text { | } \\
& \text { | } \mathrm{Rh}|\mathrm{Ti}| 1.5765 \text { | } 1.2357 \text { | } 1.5771 \text { | }
\end{aligned}
$$

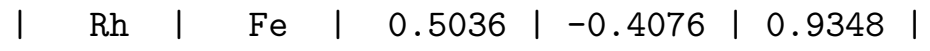

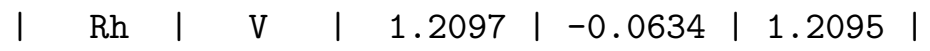

$$
\begin{aligned}
& \text { | } \mathrm{Rh} \mid \mathrm{Cr} \quad 1.2182 \text { | }-1.3584 \mid 1.9321 \text { | } \\
& \text { | Ir | Ni | } 0.8156|0.7831| 0.7751 \mid \\
& \text { | Ir | Co | } 1.2566 \mid \begin{array}{lllll}
\mid & 0.7428 & 1.2383
\end{array} \\
& \text { | Ir | Mn | } 0.1756 \text { | }-2.4545 \text { | } 1.7162 \text { | } \\
& \text { | } \operatorname{Ir}|\operatorname{Ti}| 1.5589 \text { | } 1.0883 \text { | } 1.5591 \text { | } \\
& \text { | Ir } \quad \mathrm{Fe}|0.3454|-0.5869|0.6213| \\
& \text { | Ir | V | } 1.1278|-0.1966| 1.1272 \text { | }
\end{aligned}
$$




$$
\begin{aligned}
& \text { | Ir | Cr | } 1.0547|-1.4590| 1.7315 \mid \\
& \text { | } \mathrm{Au} \text { | } \mathrm{Ni} \mid 1.8934 \text { | } 1.7712 \text { | } 1.8639 \text { | } \\
& \text { | } \mathrm{Au} \text { | Co | } 2.4579 \text { | } 1.1015 \text { | } 2.8911 \text { | } \\
& \text { | } \mathrm{Au} \quad|\quad M n \quad| \quad 1.1266 \text { | }-1.7816 \text { | } 4.4454 \text { | } \\
& \text { | } \mathrm{Au}|\mathrm{Ti}| 4.0813 \text { | None | } 4.2255 \text { | } \\
& \text { I } \mathrm{Au}|\mathrm{Fe}| 1.5256 \text { | None | } 2.5828 \text { | } \\
& \text { | } \mathrm{Au}|\mathrm{V} \quad| 3.1040 \mid 0.7602 \text { | } 4.2549 \text { | } \\
& \text { | } \mathrm{Au}|\mathrm{Cr}| 1.9448 \quad \mid-0.9947 \text { | } 4.8322 \text { | } \\
& \text { | } \mathrm{Pd}|\mathrm{Ni}| 1.3438 \text { | } 1.0052 \text { | } 1.4233 \text { | } \\
& \text { | Pd | Co | } 1.6242 \text { | None | } 2.1021 \text { | }
\end{aligned}
$$

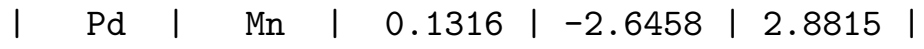

$$
\begin{aligned}
& \text { | } \mathrm{Pd}|\mathrm{Ti}| 1.6736 \text { | } 1.1416 \text { | } 1.6725 \text { | }
\end{aligned}
$$

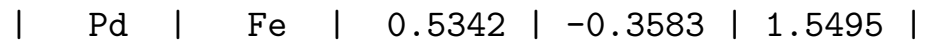

$$
\begin{aligned}
& \text { | } P d \quad|\quad V \quad| 1.6800|-0.2964| 1.8449 \text { | } \\
& \text { | } \mathrm{Pd}|\operatorname{Cr}| 1.2476 \text { | }-1.3985 \text { | } 2.8164 \mid \\
& \begin{array}{llllllllllll}
\mid & 0 s & \mathrm{Ni} & 0.5370 & 0.5218 & 0.4971 & 0.5
\end{array} \\
& \text { | Os | Co | } 0.9894 \text { | None | } 0.8710 \text { | }
\end{aligned}
$$

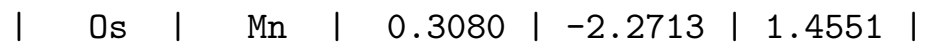

$$
\begin{aligned}
& \text { | Os | Ti | } 1.9659 \text { | } 1.2194 \text { | } 1.9666 \text { | } \\
& \begin{array}{lllllllllll}
\mid & 0 \mathrm{~s} & \mathrm{~F} & \mathrm{Fe} & 0.2452 & -0.7096 & 0.2754
\end{array}
\end{aligned}
$$

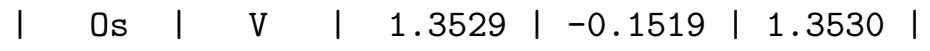

$$
\begin{aligned}
& \text { | } 0 \mathrm{~s}|\mathrm{Cr}| 1.1715 \text { | }-1.4822 \mid 1.7206 \text { | } \\
& \text { | } \mathrm{Cu}|\mathrm{Ni}| 1.8215 \text { | } 1.6771 \text { | } 1.7804 \text { | } \\
& \text { | } \mathrm{Cu} \text { | Co | } 2.4553 \text { | } 1.1005 \text { | } 2.8294 \text { | } \\
& \text { | } \mathrm{Cu} \mid \mathrm{Mn} \quad 1.3980 \text { | }-1.5153 \text { | } 4.4653 \text { | } \\
& \text { | } \mathrm{Cu}|\mathrm{Ti}| 4.3765 \text { | } 2.3705 \text { | } 4.5951 \text { | }
\end{aligned}
$$

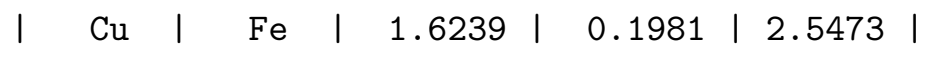

$$
\begin{aligned}
& \begin{array}{llllllllll}
\mathrm{I} & \mathrm{Cu} & \mathrm{V} & \mathrm{V} & 3.2775 & \mathbf{1} & 0.8892 & 4.4591 & \mathbf{1}
\end{array} \\
& |\mathrm{Cu}| \mathrm{Cr}|2.0953|-0.9331|4.9216|
\end{aligned}
$$




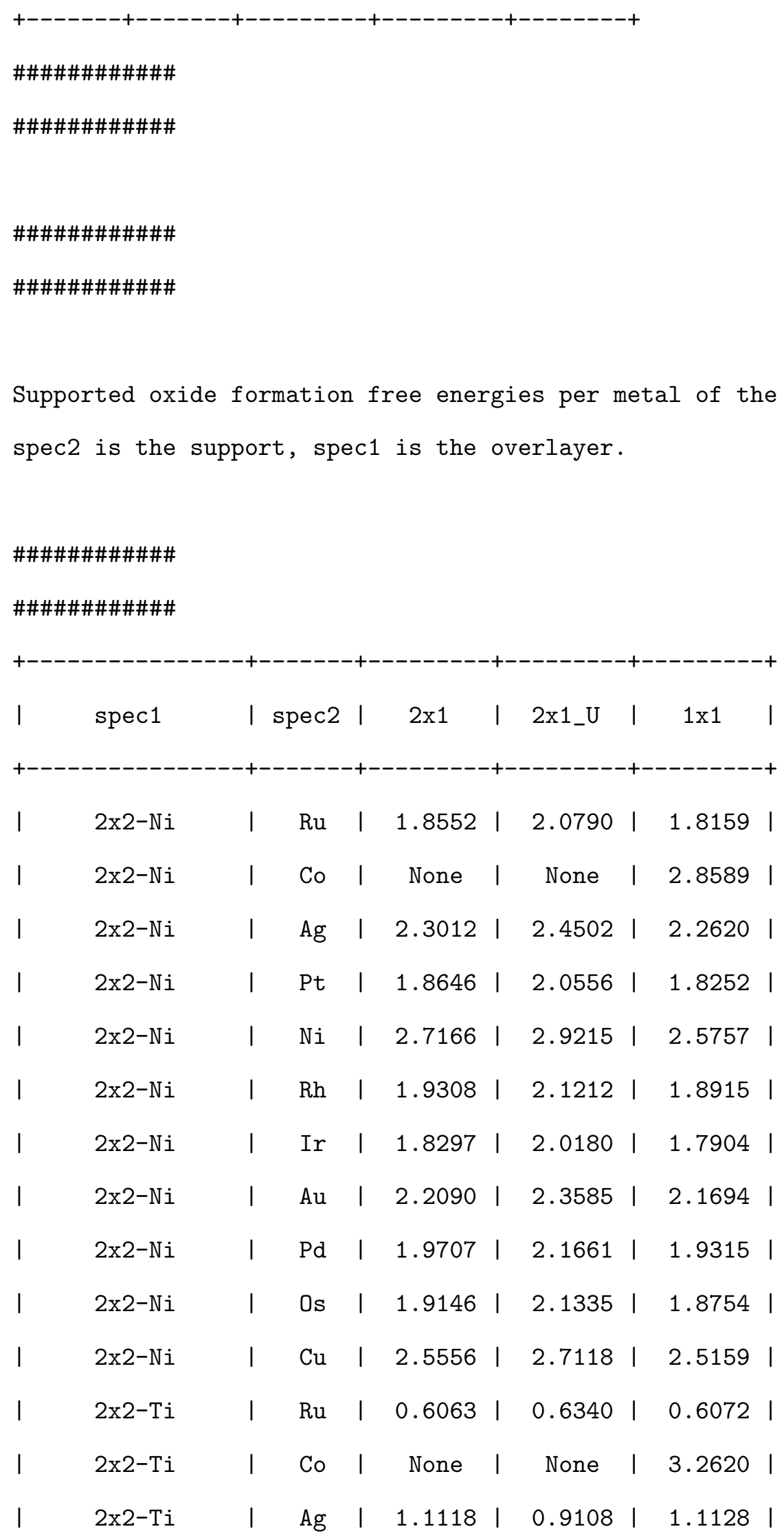




\begin{tabular}{|c|c|c|c|c|c|c|c|c|c|}
\hline & $2 \times 2-\mathrm{Ti}$ & I & Pt & I & 0.1377 & I & 0.0872 & 1 & 0.1388 \\
\hline & $2 \times 2-\mathrm{Ti}$ & 1 & $\mathrm{Ni}$ & I & 2.9073 & I & None & 1 & 2.9316 \\
\hline & $2 \times 2-\mathrm{Ti}$ & 1 & $\mathrm{Rh}$ & | & 0.7278 & I & 0.7398 & I & 0.7288 \\
\hline & $2 \times 2-\mathrm{Ti}$ & & Ir & | & 0.3709 & I & 0.3462 & I & 0.3720 \\
\hline & $2 \times 2-\mathrm{Ti}$ & & $\mathrm{Au}$ & & 0.6041 & I & 0.4219 & I & 0.6048 \\
\hline & $2 \times 2-\mathrm{T} i$ & & $\mathrm{Pd}$ & 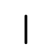 & 0.6320 & I & None & 1 & 0.6330 \\
\hline & $2 \times 2-\mathrm{Ti}$ & & Os & & 0.6949 & I & None & 1 & 0.6959 \\
\hline & $2 \times 2-\mathrm{Ti}$ & & $\mathrm{Cu}$ & I & 2.7250 & l & 2.6548 & I & 2.7648 \\
\hline & sqrt3xsqrt3-Co & I & $\mathrm{Ru}$ & 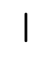 & 1.3018 & I & 1.1647 & I & 1.0708 \\
\hline & sqrt $3 x$ sqrt $3-\mathrm{Co}$ & I & $\mathrm{Ag}$ & & 2.5437 & I & None & I & 2.4681 \\
\hline & sqrt $3 \mathrm{xsqrt} 3-\mathrm{Co}$ & I & Pt & I & 1.8496 & I & 1.3556 & I & 1.9183 \\
\hline & sqrt3xsqrt3-Co & I & $\mathrm{Ni}$ & I & 1.7020 & I & 1.6870 & I & 1.4096 \\
\hline & sqrt3xsqrt3-Co & I & $\mathrm{Rh}$ & | & 1.6063 & I & 1.3545 & I & 1.4188 \\
\hline & sqrt3xsqrt3-Co & I & Ir & 1 & 1.6770 & I & 1.3198 & I & 1.4897 \\
\hline & sqrt $3 \mathrm{xsqrt} 3-\mathrm{Co}$ & I & $\mathrm{Au}$ & I & 2.5341 & I & 1.7824 & I & 2.5827 \\
\hline & sqrt $3 \mathrm{xsqrt} 3-\mathrm{Co}$ & I & $\mathrm{Pd}$ & I & 1.8531 & I & 1.4031 & I & 1.8176 \\
\hline & sqrt $3 \mathrm{xsqrt} 3-\mathrm{Co}$ & I & Os & I & 1.3143 & I & 1.1717 & I & 1.0869 \\
\hline & sqrt3xsqrt3-Co & I & $\mathrm{Cu}$ & I & 1.9645 & I & 1.7916 & I & 1.7908 \\
\hline & k-phase-Ti & I & $\mathrm{Ru}$ & 1 & -0.4198 & I & None & I & -0.4198 \\
\hline & $\mathrm{k}$-phase-Ti & I & Co & 1 & 0.4558 & I & None & I & 0.4558 \\
\hline & k-phase-Ti & 1 & $\mathrm{Ag}$ & 1 & 0.6023 & I & None & I & 0.6023 \\
\hline & $\mathrm{k}$-phase-Ti & I & Pt & I & -0.5315 & I & None & I & -0.5315 \\
\hline & $k$-phase-Ti & । & $\mathrm{Ni}$ & I & 0.3063 & I & None & I & 0.3063 \\
\hline & $\mathrm{k}$-phase-Ti & I & $\mathrm{Rh}$ & 1 & -0.1922 & । & None & 1 & -0.1922 \\
\hline & $k$-phase-Ti & I & Ir & I & -0.3055 & I & None & I & -0.3055 \\
\hline & k-phase-Ti & 1 & $\mathrm{Au}$ & I & 0.1979 & I & None & 1 & 0.1979 \\
\hline & $\mathrm{k}$-phase-Ti & I & $\mathrm{Pd}$ & I & -0.3361 & I & None & I & -0.3361 \\
\hline & $\mathrm{k}$-phase-Ti & 1 & Os & 1 & -0.1472 & I & None & & -0.1472 \\
\hline & k-phas & & & & 0.7913 & & Not & & \\
\hline
\end{tabular}




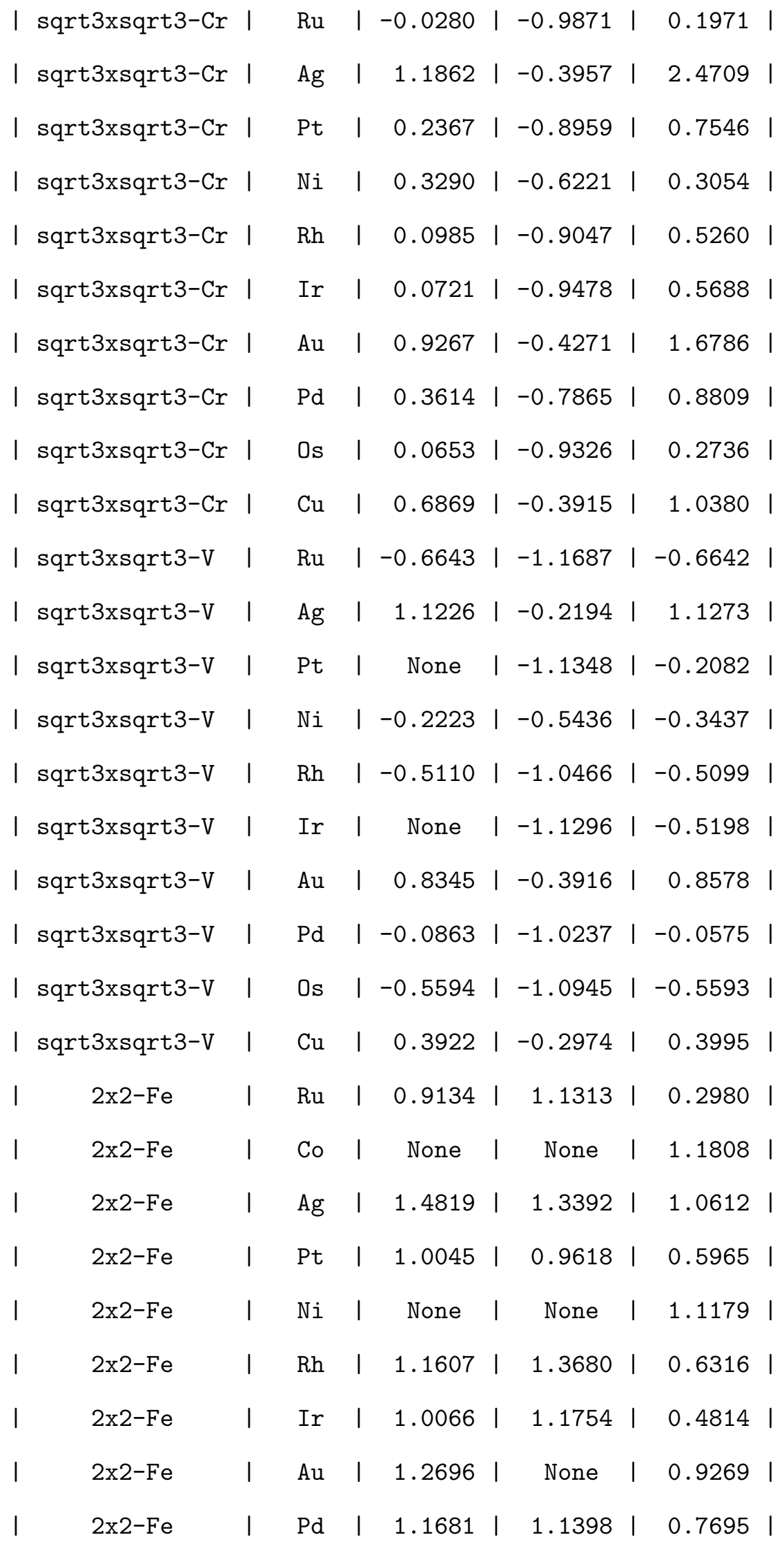




\begin{tabular}{|c|c|c|c|c|c|c|c|c|}
\hline & $2 \times 2-F e$ & 1 & Os & I & $0.9577 \quad$ & 1.2217 & 1 & 0.2971 \\
\hline & $2 \times 2-F e$ & & $\mathrm{Cu}$ & | & $1.9137 \quad$ & 2.3131 & I & 1.2195 \\
\hline & sqrt $3 x$ sqrt $3-M n$ & I & $\mathrm{Ru}$ & I & $-0.0916 \quad \mid$ & -2.0187 & I & 0.7473 \\
\hline & sqrt $3 x$ sqrt $3-M n$ & I & $\mathrm{Ag}$ & I & 0.6709 । & -1.7799 & I & 2.2842 \\
\hline & sqrt $3 \mathrm{xsqrt} 3-\mathrm{Mn}$ & I & Pt & I & $0.4983 \quad$ & -2.0751 & I & 1.3395 \\
\hline & sqrt $3 \mathrm{xsqrt} 3-\mathrm{Mn}$ & I & $\mathrm{Ni}$ & & $0.2927 \quad$ & None & 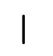 & 0.7850 \\
\hline & sqrt $3 x$ sqrt $3-M n$ & I & $\mathrm{Rh}$ & & $0.0654 \quad$ & -1.9129 & I & 0.9646 \\
\hline & sqrt3xsqrt3-Mn & 1 & Ir & I & $0.0330 \quad$ & -1.9747 & I & 0.9923 \\
\hline & sqrt3xsqrt3-Mn & I & $\mathrm{Au}$ & & None I & -1.8367 & । & None \\
\hline & sqrt $3 \mathrm{xsqrt} 3-\mathrm{Mn}$ & I & $\mathrm{Pd}$ & & 0.1067 & -2.0049 & I & 1.4119 \\
\hline & sqrt3xsqrt3-Mn & I & Os & | & -0.0105 & -1.9508 & I & 0.7985 \\
\hline & sqrt3xsqrt3-Mn & I & $\mathrm{Cu}$ & I & 0.5765 । & -1.3779 & I & 1.4076 \\
\hline & $2 \times 2-M n$ & I & $\mathrm{Ru}$ & I & $0.0153 \quad$ & -1.3379 & I & 0.2590 \\
\hline & $2 \times 2-M n$ & I & Co & & None I & None & I & 0.7105 \\
\hline & $2 \times 2-M n$ & 1 & $\mathrm{Ag}$ & & $0.6162 \mid$ & -1.2279 & I & 1.5924 \\
\hline & $2 \times 2-M n$ & I & Pt & 1 & -0.0150 & -1.6089 & 1 & 0.9065 \\
\hline & $2 \times 2-M n$ & I & $\mathrm{Ni}$ & 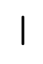 & 1.0023 & -0.0482 & I & 0.9020 \\
\hline & $2 \times 2-M n$ & I & $\mathrm{Rh}$ & I & 0.1512 & -1.2588 & I & 0.7724 \\
\hline & $2 \times 2-M n$ & 1 & Ir & I & -0.0402 & None & I & 0.6141 \\
\hline & $2 \times 2-M n$ & 1 & $\mathrm{Au}$ & I & $0.3529 \mid$ & -1.4858 & I & 1.3703 \\
\hline & $2 \times 2-M n$ & I & $\mathrm{Pd}$ & 1 & $0.2037 \quad$ & -1.4127 & I & 1.1186 \\
\hline & $2 \times 2-M n$ & 1 & Os & & $0.0890 \quad \mid$ & -1.2209 & I & 0.2245 \\
\hline & $2 \times 2-M n$ & 1 & $\mathrm{Cu}$ & I & 1.0872 & -0.1740 & I & 1.3130 \\
\hline & sqrt3xsqrt3-Ni & I & $\mathrm{Ru}$ & I & 1.1792 & 1.1772 & I & 1.1389 \\
\hline & sqrt3xsqrt3-Ni & I & $\mathrm{Ag}$ & I & 2.3507 & None & I & 2.3113 \\
\hline & sqrt3xsqrt3-Ni & I & Pt & I & 1.9769 & 2.0356 & I & 1.9524 \\
\hline & sqrt3xsqrt3-Ni & 1 & $\mathrm{Ni}$ & I & 2.1616 & None & 1 & 1.9923 \\
\hline & sqrt3xsqrt3-Ni & 1 & $\mathrm{Rh}$ & 1 & $1.5109 \mid$ & 1.5490 & I & None \\
\hline & qrt3xsqut3-Ni & & Ir & & 1.6325 & 1.6494 & & 1.5926 \\
\hline
\end{tabular}




\begin{tabular}{|c|c|c|c|c|c|c|c|c|c|}
\hline & sqrt3xsqrt3-Ni & 1 & $\mathrm{Au}$ & | & 2.5252 & & 2.6089 & I & 2.4850 \\
\hline & sqrt3xsqrt3-Ni & 1 & $\mathrm{Pd}$ & 1 & 1.8566 & I & 1.9011 & I & $1.8182 \mid$ \\
\hline & sqrt3xsqrt3-Ni & I & Os & । & 1.2378 & I & 1.2167 & I & 1.1977 \\
\hline & sqrt3xsqrt3-Ni & I & $\mathrm{Cu}$ & | & 1.7481 & I & 1.8057 & 1 & 1.7002 \\
\hline & $\mathrm{k}$-phase-V & I & $\mathrm{Ru}$ & 1 & -0.5473 & I & None & I & -0.5473 \\
\hline & k-phase-V & I & $\mathrm{Ag}$ & I & 0.8222 & I & None & 1 & 0.8222 \\
\hline & $\mathrm{k}$-phase-V & I & Pt & & -0.5279 & 1 & None & I & -0.5279 \\
\hline & $\mathrm{k}$-phase-V & I & $\mathrm{Rh}$ & & -0.4331 & I & None & I & -0.4331 \\
\hline & k-phase-V & I & Ir & 1 & -0.5444 & I & None & I & -0.5444 \\
\hline & k-phase-V & I & $\mathrm{Au}$ & 1 & 0.5299 & & None & 1 & 0.5299 \\
\hline & k-phase-V & I & $\mathrm{Pd}$ & I & -0.3409 & 1 & None & 1 & -0.3409 \\
\hline & k-phase-V & I & Os & I & -0.4716 & & None & 1 & -0.4716 \\
\hline & k-phase-V & I & $\mathrm{Cu}$ & 1 & 0.8209 & I & None & 1 & 0.8209 \\
\hline & $2 \times 2-V$ & I & $\mathrm{Ru}$ & 1 & -0.4188 & I & -0.5687 & I & $-0.4180 \mid$ \\
\hline & $2 \times 2-V$ & 1 & Co & 1 & None & & None & I & 1.0948 \\
\hline & $2 \times 2-V$ & I & $\mathrm{Ag}$ & 1 & 0.7529 & I & 0.0073 & 1 & 0.8083 \\
\hline & $2 \times 2-V$ & I & Pt & I & -0.2795 & I & -0.7551 & 1 & -0.2797 \\
\hline & $2 \times 2-V$ & I & $\mathrm{Ni}$ & I & 1.1075 & I & 1.1810 & I & 1.0065 \\
\hline & $2 \times 2-V$ & I & $\mathrm{Rh}$ & I & -0.1612 & I & -0.3267 & I & -0.1605 \\
\hline & $2 \times 2-V$ & I & Ir & I & -0.4105 & & -0.6177 & 1 & -0.4096 \\
\hline & $2 \times 2-V$ & I & $\mathrm{Au}$ & 1 & 0.3744 & 1 & -0.3894 & I & 0.4582 \\
\hline & $2 \times 2-V$ & I & $\mathrm{Pd}$ & I & 0.0618 & & -0.4268 & 1 & 0.0665 \\
\hline & $2 \times 2-V$ & I & Os & 1 & -0.3329 & & -0.4912 & I & -0.3328 \\
\hline & $2 \times 2-V$ & 1 & $\mathrm{Cu}$ & I & 1.1109 & 1 & 1.0460 & I & $1.1110 \mid$ \\
\hline & sqrt $3 x$ sqrt $3-\mathrm{Fe}$ & 1 & $\mathrm{Ru}$ & I & 0.5162 & & 0.2475 & 1 & $0.4190 \quad$ \\
\hline & sqrt $3 x$ sqrt $3-\mathrm{Fe}$ & 1 & $\mathrm{Ag}$ & I & 1.3899 & & 0.5419 & 1 & $1.7398 \mid$ \\
\hline & sqrt $3 x$ sqrt $3-F e$ & 1 & Pt & 1 & 0.7151 & 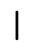 & 0.3113 & 1 & 0.9996 \\
\hline & sqrt $3 x$ sqrt $3-\mathrm{Fe}$ & 1 & $\mathrm{Ni}$ & 1 & 1.0285 & & None & I & 0.6364 \\
\hline & sqrt3xsqrt3-Fe & I & $\mathrm{Rh}$ & & 0.7685 & & 0.4120 & I & 0.6296 \\
\hline
\end{tabular}




\begin{tabular}{|c|c|c|c|c|c|c|c|c|c|}
\hline & sqrt3xsqrt3-Fe & 1 & Ir & | & 0.7246 & & 0.3498 & & 0.6753 \\
\hline & sqrt3xsqrt3-Fe & 1 & $\mathrm{Au}$ & 1 & 1.3499 & I & 0.5254 & I & 1.6267 \\
\hline & sqrt3xsqrt3-Fe & 1 & $\mathrm{Pd}$ & | & 0.7630 & I & 0.3517 & I & 0.9935 \\
\hline & sqrt3xsqrt3-Fe & I & Os & 1 & 0.5920 & 1 & 0.3253 & I & 0.4598 \\
\hline & sqrt3xsqrt3-Fe & I & $\mathrm{Cu}$ & I & 1.3398 & I & 1.0556 & I & 0.9733 \\
\hline & $2 \times 2-\mathrm{Co}$ & 1 & $\mathrm{Ru}$ & I & 1.3289 & 1 & 1.5133 & I & 1.0898 \\
\hline & $2 \times 2-\mathrm{Co}$ & I & Co & & None & I & None & 1 & 1.8906 \\
\hline & $2 \times 2-C_{0}$ & 1 & $\mathrm{Ag}$ & 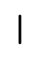 & 1.9980 & I & 1.9334 & I & 1.7587 \\
\hline & $2 \times 2-\mathrm{Co}$ & I & Pt & 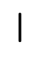 & 1.4994 & I & 1.5460 & 1 & 1.2607 \\
\hline & $2 \times 2-\mathrm{Co}$ & I & $\mathrm{Ni}$ & 1 & 2.0523 & I & 2.2173 & I & 1.7113 \\
\hline & $2 \times 2-\mathrm{Co}$ & I & $\mathrm{Rh}$ & I & 1.4206 & I & 1.5548 & 1 & 1.1818 \\
\hline & $2 \times 2-\mathrm{Co}$ & I & Ir & 1 & 1.3277 & I & None & 1 & 1.0889 \\
\hline & $2 \times 2-\mathrm{Co}$ & I & $\mathrm{Au}$ & I & 1.9049 & I & 1.7509 & I & 1.6658 \\
\hline & $2 \times 2-\mathrm{Co}$ & I & $\mathrm{Pd}$ & 1 & 1.6105 & I & 1.6897 & 1 & 1.3716 \\
\hline & $2 \times 2-\mathrm{Co}$ & I & Os & I & 1.3482 & 1 & 1.5280 & I & 1.1092 \\
\hline & $2 \times 2-\mathrm{Co}$ & I & $\mathrm{Cu}$ & 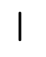 & 1.9346 & 1 & 2.0739 & I & 1.6956 \\
\hline & sqrt $3 x$ sqrt $3-\mathrm{T} i$ & 1 & $\mathrm{Ru}$ & I & -0.5725 & I & -0.7507 & 1 & -0.5748 \\
\hline & sqrt $3 x$ sqrt $3-\mathrm{T} i$ & I & $\mathrm{Ag}$ & 1 & 1.2316 & I & 0.5422 & I & 1.2327 \\
\hline & sqrt $3 x$ sqrt $3-\mathrm{T} i$ & 1 & Pt & I & -0.3820 & I & -0.5761 & I & $-0.3801 \mid$ \\
\hline & sqrt $3 x$ sqrt $3-\mathrm{T} i$ & 1 & $\mathrm{Ni}$ & 1 & 0.1298 & 1 & 0.2619 & I & 0.1586 \\
\hline & sqrt $3 x$ sqrt $3-\mathrm{T} i$ & I & $\mathrm{Rh}$ & I & -0.5366 & I & -0.6423 & I & -0.5360 \\
\hline & sqrt3xsqrt3-Ti & I & Ir & I & -0.5832 & I & -0.7240 & I & -0.5820 \\
\hline & sqrt3xsqrt3-Ti & 1 & $\mathrm{Au}$ & I & 0.8656 & I & None & I & 0.8674 \\
\hline & sqrt3xsqrt3-Ti & 1 & $\mathrm{Pd}$ & I & -0.1688 & I & -0.4563 & I & -0.1674 । \\
\hline & sqrt3xsqrt3-Ti & 1 & Os & I & -0.4469 & I & -0.6391 & 1 & $-0.4459 \mid$ \\
\hline & sqrt $3 x$ sqrt $3-\mathrm{T} i$ & I & $\mathrm{Cu}$ & 1 & 0.7948 & I & 0.5385 & I & 0.7953 \\
\hline & $2 \times 2-C r$ & I & $\mathrm{Ru}$ & 1 & -0.1078 & | & -0.6144 & 1 & -0.1054 \\
\hline & $2 \times 2-C r$ & I & Co & I & None & $\mid$ & None & I & 0.6526 \\
\hline & $2 \times 2-C r$ & | & $\mathrm{Ag}$ & & 0.8387 & 1 & None & & 1.5723 \\
\hline
\end{tabular}




\begin{tabular}{|c|c|c|c|c|c|c|}
\hline $2 \times 2-C r$ & 1 & Pt & I & $0.0630 \quad \mid$ & -0.8126 & 0.5468 \\
\hline $2 \times 2-C r$ & 1 & $\mathrm{Ni}$ & I & 1.0244 & $0.7684 \quad \mid$ & 0.9240 \\
\hline $2 \times 2-C r$ & & $\mathrm{Rh}$ & I & 0.2110 & -0.4955 & 0.4572 \\
\hline $2 \times 2-C r$ & 1 & Ir & 1 & $-0.0086 \quad \mid$ & $-0.7161 \mid$ & 0.2512 \\
\hline $2 \times 2-C r$ & I & $\mathrm{Au}$ & I & 0.5291 & -0.4938 & 1.3068 \\
\hline $2 \times 2-C r$ & I & $\mathrm{Pd}$ & I & $0.3580 \quad \mid$ & -0.5581 & 0.8242 \\
\hline $2 \times 2-C r$ & I & Os & I & $-0.0604 \quad \mid$ & -0.5434 & -0.0597 \\
\hline $2 \times 2-C r$ & I & $\mathrm{Cu}$ & I & 1.1905 & 0.6048 & 1.2581 \\
\hline
\end{tabular}

\#\#\#\#\#\#\#\#\#\#\#

\#\#\#\#\#\#\#\#\#\#\#

\section{References}

(1) Chase, M. W. J. NIST-JANAF Thermochemical Tables, 4th Edition; American Institute of Physics: New York, 1998.

(2) Lide, D. R., Ed. CRC Handbook of Chemistry and Physics, 88th Edition; CRC Press, 2007. 